\title{
Asistencia a Citología del Cuello Uterino y sus Determinantes en una Población Rural Colombiana, 1998-1999
}

Miguel A. Castro-Jiménez ${ }^{1}$, Paula A. Londoño-Cuellar ${ }^{2}$ y Lina M. Vera-Cala ${ }^{3}$

${ }^{1}$ Médico. Departamento de Salud Pública. Centro de Investigaciones Epidemiológicas. Facultad de Salud. Universidad Industrial de Santander. Bucaramanga, Colombia. E-mail: mcastro2505@yahoo.es

${ }^{2}$ Bacterióloga. Universidad Industrial de Santander. Università Degli Studi Di Milano. Milán. Italia. Email: london_p@hotmail.com

${ }^{3}$ Médica. M. Sc. Epidemiología. Departamento de Salud Pública. Facultad de Salud. Universidad Industrial de Santander. Bucaramanga. Colombia. E-mail: linamavc@yahoo.com

Recibido 12 Mayo 2006/Enviado para Modificación 9 Octubre 2006/Aceptado 30 Octubre 2006

\section{RESUMEN}

Objetivos Establecer la prevalencia de uso de la citología del cuello uterino e identificar los factores que determinan la asistencia a este examen.

Métodos Estudio de corte transversal en voluntarias de un programa de prevención de cáncer de cuello uterino realizado en Mogotes, Colombia. Se calcularon razones de prevalencia (RP). La variable dependiente fue el antecedente de asistencia a citología. Se recolectaron características sociodemográficas, clínicas, familiares y de hábitos.

Resultados La prevalencia de asistencia previa a citología fue 67,3\%. Amayor edad de la mujer aumentaba la probabilidad de tener una citología anterior. El uso de métodos de planificación familiar que requerían control (RP: 1,4; IC95\%: 1,2-1,7) y la residencia rural (RP: 0,9; IC95\%: 0,8-1,0) también fueron factores que determinaron su utilización.

Conclusiones Estos datos confirman que vivir en zonas rurales está asociado con una menor oportunidad de la mujer para acceder al examen citológico.

Palabras Clave: estudios transversales, prevalencia, frotis vaginal, accesibilidad a los servicios de salud (fuente: DeCS, BIREME)

\section{ABSTRACT}

Use and determinants of Pap smear in a rural Colombian municipality 1998-1999

Objectives Determining the prevalence of Pap smear use and identifying the factors associated with Pap test performance. 
Methods A cross-sectional study was carried out on volunteer women recruited from a local programme for detecting cervical cancer in Mogotes, Colombia. Prevalence ratios (PR) were calculated. Papanicolau smear use was the outcome being measured. Information about demographic characteristics, clinical factors, history of cancer in the family, lifestyle factors and using birth control methods was collected.

Results Pap smear use prevalence was $67,3 \%$. Age group, using birth control methods (PR:1,4; $95 \% \mathrm{Cl}: 1,2-1,7)$ and living in rural settings (PR:0,9; 95 $\% \mathrm{Cl}: 0,8-1,0)$ were the factors associated with Pap test performance.

Conclusions This data suggests that living in rural settings is associated with decreased opportunities for performing Pap tests.

Key Words: Cross-sectional study, prevalence, vaginal smear, health service accessibility (source: MeSH, NLM).

L

a disminución de la mortalidad por el cáncer invasor de cuello uterino observada en los países desarrollados fue consecuencia de la organización de programas de tamizaje que convirtieron a la citología en la herramienta fundamental de sus actividades (1-3). Sin embargo, aunque se conoce que los países menos desarrollados tienen 1,8 veces más incidencia y 2,8 veces más mortalidad por esta enfermedad, persisten algunas barreras técnicas, económicas y culturales (2) que impiden que estos programas estén establecidos o sean eficientes (4).

Aunque la citología del cuello uterino está disponible en Colombia desde hace cuatro décadas, sólo el 46 \% de las mujeres elegibles habían acudido a este examen hacia 1990 produciendo un efecto pobre sobre la mortalidad (1); en años recientes, esta cifra aumentó al 80,4 \% pero su utilización sigue siendo baja en las zonas rurales (5).

La identificación de las barreras de acceso a este examen ha sido el propósito de diferentes investigaciones basadas en poblaciones $(6,7)$ o en mujeres voluntarias (8). Durante el periodo de la investigación, no se encontraron publicaciones colombianas previas acerca de la frecuencia y los determinantes de uso del examen citológico en las mujeres que residen en municipios cuya población está distribuida principalmente fuera de la cabecera urbana.

Los objetivos de este estudio fueron determinar la prevalencia de uso de la citología del cuello uterino e identificar los factores asociados con la asistencia a este examen en mujeres de una población colombiana predominantemente 
rural. La hipótesis era que las mujeres residentes en las zonas rurales tenían menor probabilidad de tener este examen antes del estudio.

\section{MATERIALES Y MÉTODOS}

Descripción general

El estudio fue realizado en el municipio de Mogotes, Departamento de Santander, Colombia. El 65 \% de sus habitantes vivían en el área rural de la localidad durante el periodo de interés (1998-1999), por lo que el hospital local realizaba jornadas periódicas de prevención y atención clínica en estas zonas. Como complemento de estas actividades dos de los autores (Castro y Londoño) organizaron un programa de prevención del cáncer de cuello uterino en el que se motivaba a las mujeres, tanto del área rural como urbana, a asistir a la toma de la citología, se explicaba la enfermedad y se ofrecía la oportunidad de lectura del examen en una institución especializada y de tratamiento oportuno por medio de remisiones a especialistas.

Tipo de estudio y criterios de inclusión

Este es un estudio analítico de corte transversal realizado a las mujeres que participaron voluntariamente en el programa de prevención. Los criterios de inclusión al estudio fueron: tener más de 16 años y vida sexual activa.

Definición de variables

La variable dependiente (resultado) fue el antecedente de asistencia a por lo menos un examen citológico de cuello uterino antes del estudio (es decir, sí tenía una citología anterior o no la tenía). Las variables independientes fueron edad actual, lugar de nacimiento, área de residencia, menarquia, edad al inicio de las relaciones sexuales, número de compañeros sexuales, paridad, edad al primer parto, síntomas ginecológicos previos (sensación de masa pélvica, sangrado genital anormal, dolor pélvico, flujo vaginal y prurito genital), antecedentes familiares de cáncer, uso anterior o actual de métodos de planificación familiar (anticoncepción hormonal, dispositivo intrauterino, condón, método del ritmo, esterilización quirúrgica o ninguno) y, por último, se interrogó acerca del uso alguna vez en la vida de derivados del tabaco o ingesta de bebidas alcohólicas. Para la recolección de los datos se diseñó un formato que contenía las variables de interés.

Muestreo y análisis estadístico

El tamaño de la muestra se basó en las mujeres que acudieron al programa de prevención. Todas las mujeres que cumplían los criterios de inclusión 
participaron. Se realizó análisis univariado calculando media y desviación estándar (DE) para variables cuantitativas y proporciones para las categóricas. Se informa el valor mediano de variables cuantitativas en caso necesario. La asociación estadística de dos o más proporciones se estimó con la prueba de Chi al cuadrado. La base de datos fue creada en Microsoft Excel 2000 y fue analizada en STATA 7.0. La linealidad de las variables continuas se evaluó con la transformación de Box-Tidwell (9). Las razones de prevalencia (RP), intervalos de confianza al $95 \%$ (IC95\%) y valores $p$, calculados para evaluar el efecto de las variables independientes sobre el uso de citología, fueron estimados con regresión log-binomial simple y múltiple. Las cifras mayores del valor nulo (es decir, 1,0) indican una mayor probabilidad de haberse realizado la citología al menos una vez en la vida. Se siguieron los criterios de Greenland (10) para seleccionar las variables. La bondad de ajuste del modelo final se realizó con la prueba de Hosmer -Lemeshow (9).

Consideraciones éticas

Las mujeres que participaron fueron voluntarias, no hubo riesgo físico, ni psicológico. Las mujeres con resultado citológico anormal o con algún signo indicador de enfermedad en el examen físico fueron remitidas para atención especializada, lo cual ofreció una mayor probabilidad de recuperación y menor oportunidad de progresión de las lesiones. Se cumplieron los principios de la Declaración de Helsinki y de la Resolución 8430 de 1993 (11).

\section{RESULTADOS}

La edad media de las 211 mujeres participantes fue de 39,5 años (DE: 13,3años). Cerca del 70 \% residían en áreas rurales. El número mediano de embarazos fue de cuatro (rango: 0-17). Las edades medias en años durante la menarquia, el inicio de las relaciones sexuales y el primer parto fueron de 14,2 (DE: 1,6); 20,2 (DE: 5,2) y 21,9 (DE: 4,8), respectivamente.

De los síntomas indagados, 163 (77,3 \%) mujeres informaron antecedente de flujo vaginal, 131 (62,1\%) de dolor pélvico, 129 (61,1\%) de prurito, 50 $(23,7 \%)$ de sangrado genital anormal, 31 (14,7\%) de sensación de peso o masa pélvica y 186 (88,2 \%) informaron haber tenido flujo o prurito agrupados. Sólo 13 (6,2 \%) mujeres informaron ausencia de todos los síntomas.

Las prevalencias de consumo de derivados del tabaco e ingesta de bebidas alcohólicas alguna vez en la vida fueron del 6,6 \% $(n=14)$ y 17,5 \% $(n=37)$, respectivamente. La mitad de las participantes ( $\mathrm{n}=105 ; 49,8 \%$ ) habían usado métodos de planificación familiar que requerían control periódico por el personal 
de salud (dispositivo intrauterino, contraceptivos hormonales o técnica quirúrgica).

El 78,1 \% de las mujeres residentes en el área urbana y el 62,6 \% de las residentes en áreas rurales se habían realizado al menos una prueba de Papanicolaou antes de este estudio $(p<0,001)$, resultando en una prevalencia global de uso del 67,3 \%. El antecedente familiar de enfermedad neoplásica estuvo presente en 51 mujeres (24,2\%). Cerca del $70 \%$ de las mujeres participantes eran residentes del área rural (Tabla 1).

Tabla 1. Características sociodemográficas y clínicas de las mujeres incluidas en el estudio. Mogotes, Colombia, 1998-1999

\begin{tabular}{|c|c|c|c|}
\hline Variable & $\begin{array}{l}\text { Frecuencia } \\
(\mathrm{n})\end{array}$ & $\begin{array}{l}\text { frecuencia } \\
\text { relativa (\%) }\end{array}$ & $\begin{array}{c}\text { frecuencia } \\
\text { acumulada (\%) }\end{array}$ \\
\hline \multicolumn{4}{|c|}{ Edad actual (años) } \\
\hline Menor de 30 & 51 & 24,2 & 24,2 \\
\hline $30-39$ & 72 & 34,1 & 58,3 \\
\hline $40-49$ & 42 & 19,9 & 78,2 \\
\hline 50 y más & 46 & 21,8 & 100,0 \\
\hline \multicolumn{4}{|c|}{ Lugar de nacimiento } \\
\hline Mogotes & 173 & 82,0 & 82,0 \\
\hline Otro municipio & 38 & 18,0 & 100,0 \\
\hline \multicolumn{4}{|c|}{ Zona de residencia } \\
\hline Urbana & 64 & 30,3 & 30,3 \\
\hline Rural $^{a}$ & 147 & 69,7 & 100,0 \\
\hline \multicolumn{4}{|l|}{ Gestaciones } \\
\hline Ninguna & 11 & 5,2 & 5,2 \\
\hline Una a cinco & 131 & 62,1 & 67,3 \\
\hline Seis y más & 69 & 32,7 & 100,0 \\
\hline \multicolumn{4}{|c|}{ Edad al primer parto } \\
\hline Menos de 20 & 79 & 39,5 & 39,5 \\
\hline $20-29$ & 106 & 53,0 & 92,5 \\
\hline 30 y más & 15 & 7,5 & 100,0 \\
\hline
\end{tabular}

El análisis bivariado de las características sociodemográficas muestra que a mayor edad de la mujer existía mayor probabilidad de asistencia previa al examen citológico; no obstante, se observa que en las de 50 y más años esta probabilidad era 20 \% menor que en las del intervalo anterior. Las residentes en áreas rurales y las nacidas fuera del municipio de Mogotes tenían menor probabilidad puntual de tener el examen, aunque sus asociaciones no fueron significativas (Tabla 2).

En cuanto a las características clínicas, el análisis bivariado mostró que la probabilidad de tener una citología previa el estudio era mayor en las mujeres con uno a cinco embarazos (RP: 2,5; IC95\%: 0,9-6,6) o con más de cinco 
embarazos (RP: 2,7; IC95 \%: 1,0-7,0) que en aquellas que nunca habían estado embarazadas (RP: 1,0). Esta probabilidad también aumentaba en forma significativa con el antecedente de prurito genital (RP: 1,3; IC95\%: 1,0-1,6) y el uso de métodos de planificación que requerían control periódico (RP: 1,4; IC95 \%: 1,2-1,7). Aunque las mujeres con antecedente de dolor pélvico (RP: 1,1; IC95 \%: 0,9-1,3) y flujo vaginal (RP: 1,2; IC95 \%: 0,9-1,6) tenían una mayor probabilidad puntual de uso de citología anterior, estas asociaciones no fueron significativas. Los síntomas prurito genital y flujo vaginal también se agruparon, pero no demostraron tener efecto durante el análisis bivariado (RP: 1,2; IC95 \%: 0,8-1,8; p = 0,263). Los demás antecedentes clínicos no mostraron asociación con la variable de salida.

Tabla 2. Antecedente de citología según características sociodemográficas. Mogotes, Colombia, 1998-1999. Regresión binomial bivariada

\begin{tabular}{lccc}
\hline \multicolumn{1}{c}{ Variable } & $\begin{array}{c}\text { Razón de } \\
\text { prevalencia }\end{array}$ & IC95 \% ${ }^{\text {a }}$ & Valor $p$ \\
\hline Edad actual (años) & 1,0 & & \\
Menor de 30 & 1,5 & $1,1-2,0$ & 0,016 \\
30-39 & 1,6 & $1,2-2,3$ & 0,002 \\
$40-49$ & 1,4 & $1,0-1,9$ & 0,007 \\
\hline 50 y más & 1,0 & & \\
\hline Lugar de nacimiento & 0,9 & $0,7-1,1$ & 0,316 \\
\hline Mogotes & & & \\
Otro municipio & 1,0 & & \\
\hline Zona de residencia & 0,8 & $0,6-0,9$ & \\
Urbana & & & \\
Rural & b
\end{tabular}

a. IC95\%: intervalo de confianza al $95 \%$; b. se incluyen dos mujeres residentes en un municipio diferente al de estudio

La historia familiar de cáncer (RP: 0,9; IC95 \%: 0,7 - 1,2) y los consumos, alguna vez en la vida, de alcohol (RP: 1,1; IC95 \%: 0,9 - 1,4) y de productos derivados del tabaco (RP: 1,2; IC95\%: 0,9-1,6) no estuvieron asociados estadísticamente con la asistencia anterior a la citología.

Las variables que persistieron en el modelo final luego de ajustar por los demás factores y que, por tanto, alteran de manera independiente la probabilidad de uso del examen citológico de cuello uterino son: la edad de la mujer, el uso de métodos de planificación y la zona de residencia en el municipio (Tabla 3). La bondad de ajuste mostró que el modelo obtenido describe apropiadamente los datos $\left(\mathrm{Chi}^{2}=4,96 ; \mathrm{p}=0,66\right)$. 
254 REVISTA DE SALUD PÚBLICA · Volumen 8 (3), Noviembre 2006

Tabla 3. Determinantes del uso anterior del examen citológico de cuello uterino. Mogotes, Colombia, 1998-1999. Modelo final. Regresión binomial múltiple

\begin{tabular}{|c|c|c|}
\hline Variable & $\begin{array}{c}\text { Razón de } \\
\text { prevalencia }\end{array}$ & $1 C 95 \%{ }^{a}$ \\
\hline \multicolumn{3}{|l|}{ Edad actual (años) } \\
\hline Menor de 30 & 1,0 & \\
\hline $30-39$ & 1,4 & $1,1-1,9$ \\
\hline $40-49$ & 1,6 & $1,2-2,1$ \\
\hline 50 y más & 1,6 & $1,2-2,1$ \\
\hline \multicolumn{3}{|l|}{ Zona de residencia } \\
\hline Urbana & 1,0 & \\
\hline Rural $^{b}$ & 0,9 & $0,8-1,0$ \\
\hline \multicolumn{3}{|l|}{ Método de PLF ${ }^{\mathrm{C}}$} \\
\hline $\begin{array}{l}\text { Ninguno/ no requiere } \\
\text { citas }\end{array}$ & 1,0 & \\
\hline $\begin{array}{l}\text { Requiere control } \\
\text { regular }\end{array}$ & 1,4 & $1,2-1,7$ \\
\hline
\end{tabular}

\section{DISCUSIÓN}

Sólo el 67,3 \% de las mujeres informaron tener al menos un examen citológico antes de su participación en este estudio. Una limitación de esta investigación es la condición de voluntarias de las mujeres debido a que es posible que estas participantes tengan una mejor actitud hacia los servicios de salud y, por tanto, la prevalencia de uso podría ser mayor en este grupo que en las mujeres de la población general. No obstante, bajo esta premisa es preocupante que la proporción encontrada no sea mayor ya que estaría indicando la existencia de una dificultad real de acceso a la citología en las mujeres residentes en municipios de predominio rural y que puede existir un porcentaje importante de mujeres que desean realizarse este examen pero en quienes existe alguna barrera que no se los permite (por ejemplo, algunas razones expresadas por las mujeres, aunque no fueron cuantificadas, son las condiciones económicas, la distancia al centro de salud, la deficiencia en medios de transporte hacia la cabecera municipal y la falta de educación en la pareja).

En este estudio, las mujeres que eran residentes en áreas rurales tuvieron una probabilidad significativamente más baja de haber tenido una citología previa que las de la zona urbana, lo cual también fue descrito por Claeys (6) y apoya la hipótesis de esta investigación referente a que vivir fuera de la cabecera urbana es un factor que limita el acceso de las mujeres a este examen. A mayor edad de la entrevistada también se encontró una mayor probabilidad de haberse realizado la citología. Este efecto de la edad es plausible y también es consistente con 
otros estudios (6,7,12). Maxwell (13) encontró que las mujeres mayores de 60 años tenían $47 \%$ menos probabilidad de haberla usado que las de 30 a 54, teniendo un comportamiento similar a lo observado en esta investigación cuando se analizó el efecto crudo de edad sobre la frecuencia de uso, aunque desapareció luego de ajustar por las demás variables.

Una posible fuente de mala clasificación en este estudio es que debido a tabúes en torno a la sexualidad pudo existir una tendencia de las mujeres a referir un menor número de compañeros sexuales, aunque posiblemente sea no diferencial y, por tanto, haya afectado por igual a usuarias y no usuarias del examen. La probabilidad de que una mujer se hubiera tomado el examen citológico con anterioridad aumentaba en un $4 \%$ con respecto a la que había tenido una pareja sexual menos, no obstante, esta variable fue descartada por los análisis durante la obtención del modelo final.

Nascimento (7) informó que las mujeres que usaban anticoncepción hormonal tenían 2,1 veces mayor probabilidad de haber asistido a una citología, similar a lo que se ha informado en este estudio, no obstante, hay que tener en cuenta que en el presente estudio este método fue agrupado con otros que también requerían algún control regular por parte del personal de salud.

La paridad fue asociada al uso de la prueba de Papanicolaou en un estudio previo (12) similar a lo publicado en este informe durante el análisis bivariado, sin embargo, aunque existe una razón de prevalencia puntual a favor de las mujeres que tuvieron al menos un embarazo, no se encontró un efecto independiente. Dos factores que se han asociado al uso de la citología pero que en esta investigación no tuvieron efecto independiente fueron la historia familiar de cáncer (12) y el lugar de nacimiento (7). En este estudio no se encontró una mayor prevalencia de citología en mujeres con antecedente de consumo de bebidas alcohólicas o de productos derivados del tabaco. Aunque el prurito genital y el flujo vaginal cumplieron los criterios de selección durante el análisis bivariado tampoco permanecieron en el modelo final. No se encontraron publicaciones que estudiaran la influencia de los síntomas ginecológicos sobre el uso de esta prueba.

Algunas variables que pueden estar asociadas al uso de este examen como los ingresos del hogar (6), el estado marital (7), la tenencia de seguridad social (14), la asistencia a consulta en el año anterior (15) y las creencias y actitudes de la mujer hacia la enfermedad (16) no fueron preguntadas. 
Finalmente, con base en estos hallazgos, los autores recomiendan el desplazamiento de equipos interdisciplinarios a los puestos de salud rurales como parte de los programas de tamizaje oficiales, al menos, mientras se realizan los ajustes necesarios para garantizar un acceso fácil, oportuno y permanente que beneficie a las mujeres que viven en zonas lejanas de los hospitales locales y que permitan cumplir también con el segundo objetivo de estos programas: realizar el tratamiento temprano a las mujeres que lo necesiten.

Agradecimientos. A las mujeres que participaron en el Programa de Prevención de Cáncer de Cuello Uterino; a la auxiliar de laboratorio Maria Luisa Figueroa López y a las auxiliares de enfermería pertenecientes al Hospital Integrado San Pedro Claver de Mogotes, a la trabajadora social Janeth Rocío Díaz Cadena y a las citotecnólogas de la Liga Santandereana de Lucha contra el Cáncer por la capacitación ofrecida en toma de muestras.

\section{REFERENCIAS}

1. Sankaranarayanan R, Budukh AM, Rajkumar R. Effective screening programmes for cervical cancer in low- and middle- income developing countries. Bull World Health Org 2001; 79 (10): 954-961.

2. Chirenje ZM, Rusakaniko S, Kirumbi L, Ngwalle EW, Makuta-Tlebere P, Kaggwa S, et al. Situation analysis for cervical cancer diagnosis and treatment in East, Central and Southern Africa Countries. Bull World Health Org 2001; 79 (2): 127-132.

3. Valdespino VM, Valdespino VE. Cervical Cancer Screening: State of the Art. Curr Opin Obstet Gynecol 2005; 18: 35-40.

4. Ferlay J, Bray FJ, Bray F, Pisani P, Parkin DM. GLOBOCAN 2002: Cancer Incidence, Mortality and Prevalence Worldwide. IARC Cancer Base No. 5, version 2.0. Lyon: IARC Press; 2004.

5. Ministerio de Salud de Colombia. Estudio Nacional de Factores de Riesgo de Enfermedades Crónicas (ENFREC II). Serie: Conocimientos, Actitudes y Prácticas en Detección temprana de Cáncer de Cervico Uterino y de Mama; 1999.

6. Claeys P, Gonzalez C, Gonzalez M, Page H, Bello RE, Temmerman M. Determinants of cervical cancer screening in a poor area: results of a population-based survey in Rivas, Nicaragua. Trop Med Int Health 2002; 7(11): 935-941.

7. Nascimento CMR, Eluf-Neto J, Rego RA. Cobertura do teste de Papanicolaou no municipio de São Paulo e características das mulheres que realizaram o teste. Bol Oficina Sanit Panam 1996; 121 (6): 491-501. 
8. Aaron DJ, Markovic N, Danielson ME, Honnold JA, Janosky JE, Schmidt NJ. Behavioral Risk Factors for Disease and Preventive Health Practices Among Lesbians. Am J Public Health 2001; 91(6): 972-975.

9. Hosmer DW, Lemeshow S. Applied Logistic Regression. NewYork: A WilleyInterscience Publication;1989.

10. Greenland S. Modeling and variable selection in Epidemiologic Analysis. Am J Public Health 1989; 79: 340-349.

11. Ministerio de Salud de Colombia. Resolución 8430 de octubre de 1993. Bogotá; 1993.

12. Klimovsky E, Matos E. El uso de la prueba de Papanicolaou por una población de Buenos Aires. Bol Oficina Sanit Panam 1996; 121 (6): 502-509.

13. Maxwell JC, Bancej CM, Snider J, Vik S. Factors Important in Promoting Cervical Cancer Screening among Canadian Women: Findings from the 1996-97 National Population Health Survey (NPHS). Can J Public Health 2001; 92 (2): $127-133$

14. Dias da Costa JS, Berenhauser P, Manzolli P, Moreira MR. Cobertura do exame histopatológico na cidade de Pelotas, Brasil. Rev Panam Salud Publica 1998; 3(5): 308-313.

15. O’Malley AS, Mandelblatt J, Gold K, Cagney KA, Kerner J. Continuity of Care and the Use of Breast and Cervical Screening Services in a Multiethnic Community. Arch Intern Med 1997; 157(13): 1462-1470.

16. Hubbell FA, Chavez LR, Mishra SI, Valdez RB. Beliefs About Sexual Behavior and Other Predictors of Papanicolaou Smear Screening Among Latinas and Anglo Women. Arch Intern Med. 1996; 156(20):2353-2358. 